\title{
World Journal of Incidence of pediatric injury visits
Pediatric Surgery decreased while trauma surgeries remained stable during the first and second waves of the COVID-19 pandemic in Finland: a register-based study
}

Joonas Kuorikoski (D) , ${ }^{1}$ Ilari Kuitunen, ${ }^{2,3}$ Mikko Uimonen, ${ }^{1}$ Ville M Mattila, ${ }^{4,5}$ Ville Ponkilainen ${ }^{1}$

To cite: Kuorikoski J, Kuitunen I, Uimonen $\mathrm{M}$, et al. Incidence of pediatric injury visits decreased while trauma surgeries remained stable during the first and second waves of the COVID-19 pandemic in Finland: a register-based study. World Jnl Ped Surgery 2021;4:e000304. doi:10.1136/ wjps-2021-000304

Received 2 May 2021 Accepted 13 September 2021

Check for updates

(c) Author(s) (or their employer(s)) 2021. Re-use permitted under CC BY-NC. No commercial re-use. See rights and permissions. Published by BMJ.

${ }^{1}$ Department of Surgery, Central Finland Central Hospital, Jyvaskyla, Finland

2Department of Pediatrics,

Mikkeli Central Hospital, Mikkeli, Finland

${ }^{3}$ Department of Pediatrics, University of Eastern Finland, Mikkeli, Finland

${ }^{4}$ Department of Orthopaedics and Traumatology, Tampere University Hospital, Tampere, Finland

${ }^{5}$ Faculty of Medicine and Health Technology, Tampere University, Tampere, Finland

Correspondence to Dr Joonas Kuorikoski; joonas. kuorikoski@fimnet.fi

\section{ABSTRACT}

Introduction The COVID-19 pandemic has reduced pediatric emergency department (ED) visits and surgeries. This study evaluates the incidence of pediatric trauma ED visits and surgeries in Finland during the first and second waves of the pandemic.

Methods Three large Finnish hospitals, covering one-sixth of the Finnish pediatric population, participated. Data on all ED visits and trauma surgeries between January 2017 and December 2020 were collected from hospital discharge registers. Monthly incidences with $95 \% \mathrm{Cl}$ were calculated per 100000 person-months by Poisson exact method and compared by incidence rate ratio (IRR).

Results During the lockdown the incidence of head injuries (IRR $0.19,95 \% \mathrm{Cl} 0.04$ to 0.87 ), sprains (IRR $0.25,95 \% \mathrm{Cl} 0.14$ to 0.46 ), and fractures (IRR $0.36,95 \% \mathrm{Cl}$ 0.25 to 0.51 ) decreased in the 13-17 years age group. In the $4-12$ years age group a $55 \%$ decrease (IRR $0.45,95 \%$ $\mathrm{Cl} 0.22$ to 0.96 ) in head injuries was observed. During the period of regional restrictions a subtle decrease in head injuries was seen in the 13-17 years age group (IRR $0.26,95 \% \mathrm{Cl} 0.09$ to 0.78 ). During the lockdown in March 2020 the incidence of fractures decreased in the oldest age group (13-17 years) (IRR $0.62,95 \% \mathrm{Cl} 0.46$ to 0.85 ), while a rebound in incidence was seen at the end of the lockdown period in June.

Conclusion The nationwide lockdown and the cancellation of sports and other hobbies markedly decreased the injuries among children aged $13-17$ years, while the decrease was lower among children aged 4-12 years. Cancellation of sports and hobbies did not affect patients under 4 years of age.

\section{INTRODUCTION}

The COVID-19 pandemic caused by SARSCoV-2 has affected healthcare systems all over the world and the battle against the virus continues. ${ }^{1}$ Moreover, the indirect effect of the pandemic on the pediatric population may be substantial. ${ }^{2}$ On March 16, 2020 , the Finnish Government declared a state of emergency and a nationwide lockdown was
Key messages

What is already known about this subject?

- Emergency department visits due to trauma decreased during the COVID-19 pandemic.

- The COVID-19 pandemic may have substantial indirect effects on the pediatric population.

- Changes in injury patterns have been observed during the pandemic.

What are the new findings?

- The lockdown influences the incidence of pediatric trauma visits.

- The incidence of trauma visits decreased among patients older than 4 years.

- This change can be explained by the cancellation of sports and other hobbies.

How might it impact on clinical practice in the foreseeable future?

- The study implies that during possible future lockdowns, healthcare assets can be retargeted from pediatric trauma to treatment of the pandemic.

initiated. Schools were closed and teaching was arranged remotely. In addition, public gatherings were limited and people were advised not to spend time in public places. All national and municipal hobby and leisure centers, sports facilities, and youth centers were closed. ${ }^{3}$ The lockdown was lifted in May after a rapid decrease in incidence of COVID-19 cases. ${ }^{4}$ Even though the COVID-19 pandemic again worsened in autumn of 2020, no lockdown was ordered and regional restrictions were applied instead. ${ }^{4}$ A major goal during the second wave of COVID-19 cases in Finland was to mitigate the impact of the restrictions on children and therefore schools and day care centers remained open. ${ }^{5}$

In the Finnish population, the COVID-19 pandemic has reduced the number of 
emergency department (ED) visits. ${ }^{6} 7$ Indeed, similar trends regarding acute pediatric trauma visits and operations have also been reported in other countries. ${ }^{8-13} \mathrm{~A}$ previous Finnish study showed that the rate of severely injured patients remained stable during the pandemic. ${ }^{14}$ Parallel results were reported when the pandemic also increased referrals to major trauma centers in the pediatric population. ${ }^{9}$ A previous study from Finland reported a decrease in incidence of pediatric trauma operations during the lockdown, mostly due to a decreased rate of lower limb trauma operations. Interestingly, a clear decrease in day care, school, and organized sports-related injuries was also reported, although minor injuries were not recorded. ${ }^{15}$

Changes in injury patterns have been observed during the pandemic. In a recent study the incidence of blunt trauma cases decreased, whereas the incidence of penetrating and burn injuries remained stable. ${ }^{16}$ In addition, a decrease in the incidence of sports-related injuries has been reported. ${ }^{911} 1316$ Injuries occurring at home or on bicycles, in turn, have increased. ${ }^{11}$

This study evaluates the incidence of pediatric trauma in Finland during the first and second waves of the COVID-19 pandemic and assesses the impact of national lockdown and regional restrictions on the incidence of pediatric trauma ED visits and surgeries.

\section{METHODS}

\section{Study setting and patients}

The study was conducted in three large Finnish hospitals (Tampere University Hospital, Mikkeli Central Hospital, and Central Finland Hospital) with a catchment area covering a pediatric population of 170000 inhabitants (one-sixth of the Finnish pediatric population). ${ }^{17}$ Mikkeli Central Hospital and Central Finland Hospital each has a combined primary and secondary level ED, and both hospitals serve as regional secondary level trauma units. Tampere University Hospital is a tertiary level trauma unit. These hospitals provide specialized healthcare services required in treating injured children. The study period was from January 2017 to December 2020. The number of ED visits due to pediatric trauma was collected from the patient information systems of the participating hospitals using the Finnish version of the International Classification of Diseases 10th Revision (ICD-10) diagnostic codes for injuries. The codes (all codes beginning with S: injuries) were classified based on the second digit of the code, which indicates the severity of the injury. Codes with 3 as the second digit (sprains and dislocations) were considered as sprains and dislocations. Codes with 2 as the second digit were considered as fractures. Codes beginning with S06.* were considered as head injuries.

The number of trauma surgeries was collected from the electronic medical record systems of the participating hospitals using the Nordic Medico-Statistical Committee (NOMESCO) Classification of Surgical Procedures codes
(Finnish version). Procedure codes beginning with $\mathrm{N}^{*}$ (musculoskeletal operations) were included as fracture surgery. All elective operations were excluded.

The primary outcome was the incidence of ED visits due to pediatric trauma during the first and second waves of the COVID-19 pandemic in Finland. The secondary outcome was the incidence of pediatric trauma surgeries performed during the COVID-19 pandemic in comparison with the normal situation in the reference years.

\section{Statistical analysis}

We divided patients into the following three age groups: 0-3 years, 4-12 years, and 13-17 years. The monthly incidences of ED visits due to pediatric trauma and pediatric trauma surgery in the three participating hospitals were then calculated for each age group. We compared the months in 2020 with the mean incidence of the corresponding months during the years 2017-2019. Monthly incidence was computed by dividing the number of injuries and operations per month by the population size of the corresponding age group. The yearly mean population size of the three hospital districts was used (approximately 175000 persons). Monthly incidences with 95\% CI were calculated per 100000 personmonths by the Poisson exact method and were compared by incidence rate ratio (IRR). Analyses were performed using R V.4.0.2 (R Foundation for Statistical Computing, Vienna, Austria).

\section{RESULTS}

\section{Head injuries}

Head injury was diagnosed in 305 cases during the pandemic year, which was $14.1 \%$ fewer than the average in the previous years ( $\mathrm{n}=355$ cases annually). During the lockdown period a decrease in the incidence of head injuries was observed in April in the 4-12 years (IRR $0.45,95 \%$ CI 0.22 to 0.96 ) and the $13-17$ years (IRR 0.19 , $95 \%$ CI 0.04 to 0.87 ) age groups. During the regional restrictions in October the incidence decreased in the $13-17$ years age group (IRR $0.26,95 \%$ CI 0.09 to 0.78 ), while remaining stable in the $0-3$ years and in the $4-12$ years age groups (figure 1 ).

\section{Fractures}

The initial lockdown period had no effect on the incidence of fracture-related visits in the 0-3 years or in the 4-12 years age groups. During the first months of 2020 and during the lockdown in March 2020, the incidence of fractures decreased in the oldest age group (13-17 years) (IRR $0.62,95 \%$ CI 0.46 to 0.85 ), while a rebound in incidence was seen at the end of the lockdown period in June. After the lockdown, the incidence of fracturerelated visits stayed slightly elevated until October (IRR $1.46,95 \%$ CI 1.04 to 2.04 ) in the $4-12$ years age group (figure 2).

\section{Sprains and dislocations}

In 2020, 1211 ED visits occurred due to minor pediatric injuries, whereas the average in the reference years was 


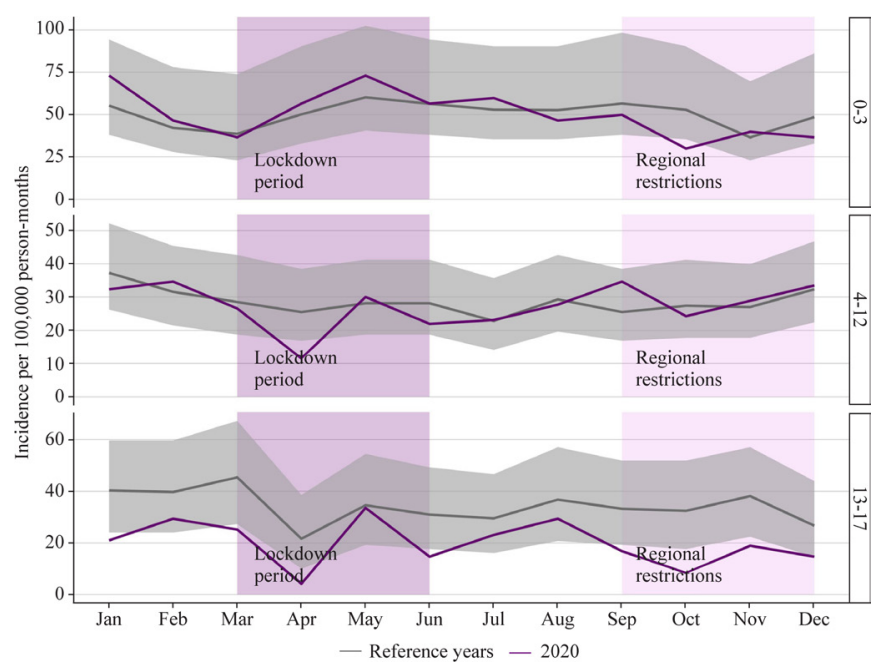

Figure 1 Incidence of pediatric head injuries in 2020 compared with reference years (2017-2019).

1351 visits, resulting in a $10 \%$ annual decrease in visits. The incidence of minor injuries was also lower during the pandemic compared with the previous years. This decrease was most notable during the lockdown in April in the 4-12 years age group (IRR $0.46,95 \%$ CI 0.28 to 0.76 ) and in the $13-17$ years age group (IRR $0.25,95 \% \mathrm{CI}$ 0.14 to 0.46$)$. The incidence of minor injuries remained stable during the regional restrictions in all age groups (figure 3).

\section{Operations}

No notable changes were seen in the incidence of pediatric trauma surgery operations due to the COVID-19 pandemic in any age group. A total of 356 operations were performed during the COVID-19 pandemic year, whereas the annual average was 360 during the reference years (figure 4 ).

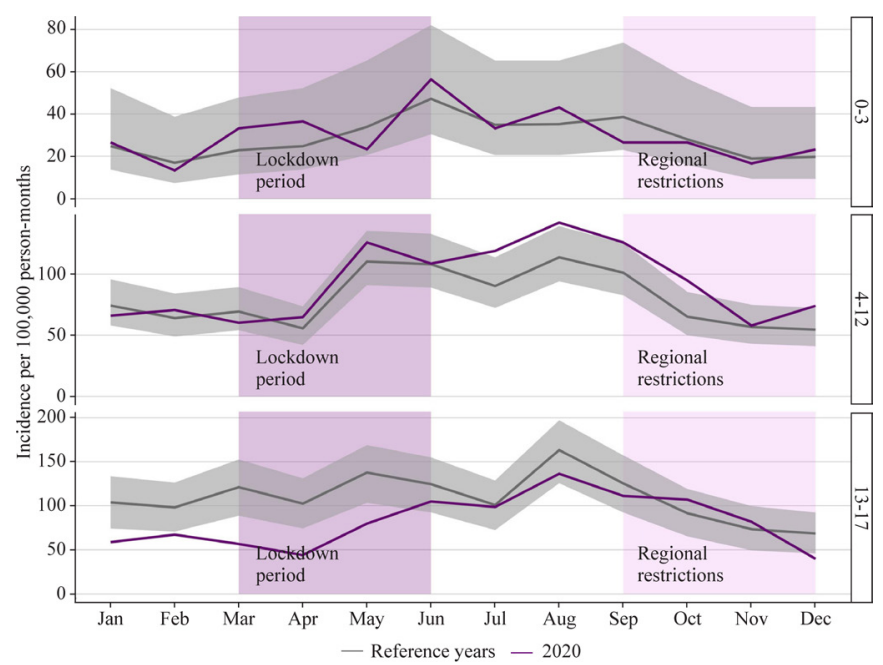

Figure 2 Incidence of pediatric fractures in 2020 compared with reference years (2017-2019).

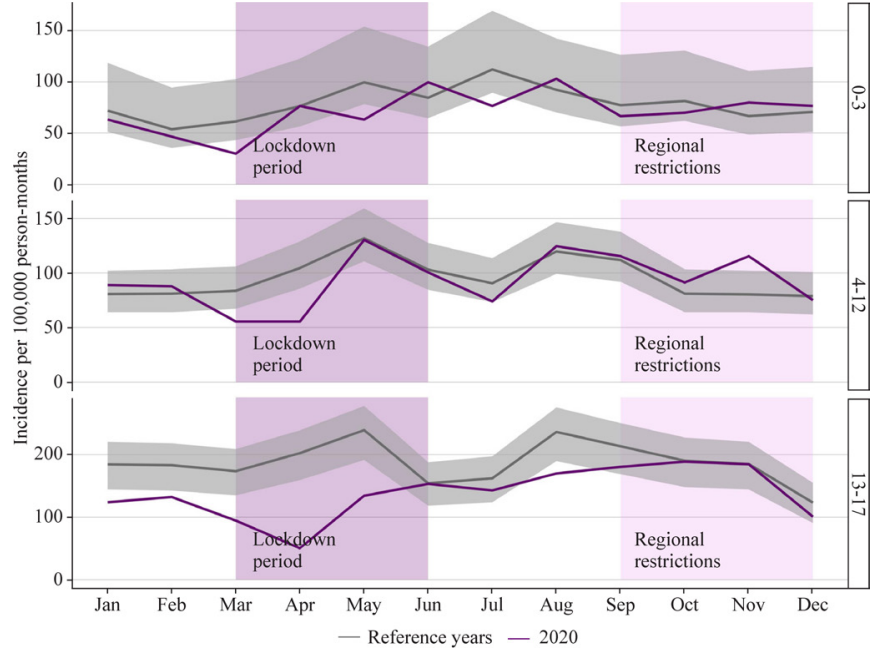

Figure 3 Incidence of pediatric sprains and dislocations in 2020 compared with reference years (2017-2019).

\section{DISCUSSION}

The COVID-19 pandemic reduced the annual rate of pediatric trauma visits by $10 \%$ in 2020 . Moreover, during the first wave of the COVID-19 pandemic, the lockdown had a subtle but clear impact on the number of pediatric trauma visits in certain age groups. For example, the incidence of head injuries, sprains, and fractures decreased during the lockdown in the oldest age group (13-17 years), whereas the lockdown did not affect the rate of these injuries in the younger age groups. Interestingly, during the lockdown, the rate of ED visits due to sprains first decreased in the 4-12 years age group and then increased back to normal levels in May. A similar pattern was seen in the incidence of sprains in the 13-17 years age group.

During the regional restrictions period, a subtle decrease in head injuries was observed in the $0-3$ years and in the 13-17 years age groups. Interestingly, during regional restrictions the rate of fractures in the youngest

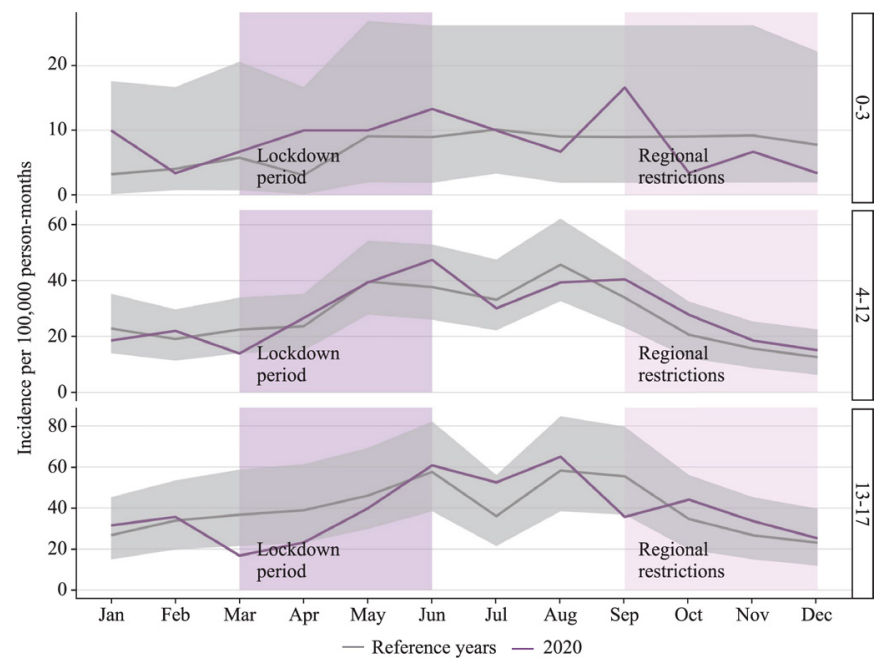

Figure 4 Incidence of pediatric trauma operations in 2020 compared with reference years (2017-2019). 
and oldest age groups remained stable, but an increase was seen in the 4-12 years age group.

An increase in minor injuries during the COVID-19 pandemic has been reported in a previous study. ${ }^{9}$ In the current study, however, we did not have a similar finding. Even though more time spent at home and the lack of highvelocity sport events might protect against more severe injuries, such as fractures, it is possible that children will seek new activities to fill the gap left by the ban on organized sports. Indeed, Baxter et af reported an increase in the incidence of rollerblade, scooter, bicycle, and skateboard injuries, although these injuries were mostly minor. It is possible that these injuries are so minor that these patients do not seek medical care at all, which might explain, at least partly, the differing results found in our study.

The incidence of head injury was lower in 2020 than in the reference years, with a $14.1 \%$ decrease in the number of cases. During the lockdown period a decrease was seen in the incidence of head injuries in the older age groups (4-12 years and 13-17 years), whereas the incidence remained stable in the youngest age group (0-3 years). During the regional restrictions a decrease in the incidence of head injury was seen in the 13-17 years age group, whereas the incidence in the 0-3 and 4-12 years age groups remained stable. This is an interesting finding because fall is a common injury mechanism for minor blunt head trauma patients in the pediatric population. ${ }^{18}$

In a Swedish epidemiological study the most common injury mechanism for pediatric fractures was sports and play. ${ }^{19}$ Rennie et $a t^{20}$ reported fall to be the most common cause of pediatric fracture. A decrease in sports injuries during the COVID-19 pandemic has previously been described. ${ }^{911} 1316$ In addition, a recent study showed that the COVID-19 pandemic has had a clear impact on injury mechanism distribution in pediatric trauma. ${ }^{16}$ Although we did not directly study injury mechanisms, our results suggest that the injury mechanisms in the pediatric trauma population of our study also changed during the pandemic because the rates of head injuries, fractures, and sprains all decreased in certain age groups. All national and municipal sporting activities were canceled during the lockdown, which might explain our results. During the lockdown in April schools were closed and organized activities were postponed, resulting in a decrease in the incidence of injuries.

Unlike previous studies, we did not see a clear decrease in pediatric trauma operations during the COVID-19 pandemic. ${ }^{89}$ Indeed, we only saw a small decrease in the number of operations in the oldest age group (13-17 years) in March. The decrease in the oldest age group was probably due to the suspension of leisure-time activities, resulting in fewer severe injuries.

In our study we had access to a large database formed from three different hospitals covering all levels of care from primary to tertiary. In Finland it is mandatory that the ICD-10 diagnosis code is added to a patient's records after an ED visit and an operation code is added after an operation. Hence, the data used in this study were robust. The multicenter study design involving three large hospitals with a homogeneous patient population improves the generalizability of the study findings to the Finnish pediatric population. One of the major weaknesses of this study was the lack of information on injury mechanism, which prevented a more sophisticated elaboration of the changes in the incidence of ED visits and surgery for trauma in our study population.

In conclusion, the annual rate of pediatric trauma visits decreased during the COVID-19 pandemic. During the nationwide lockdown the incidence of head injuries and sprains decreased in the 4-12 years and in the 13-17 years age groups, while the incidence of fractures decreased only in the 13-17 years age group. The incidence of pediatric trauma surgery remained stable in 2020.

Twitter Ilari Kuitunen @ilarikuitunen

Acknowledgements We would like to thank Peter Heath for revising the language of this manuscript.

Contributors JK contributed to writing - original draft, data curation, formal analysis. IK, MU contributed to data curation, writing - review and editing. VMM contributed to conceptualisation, data curation, validation, writing - review and editing. VP contributed to data curation, formal analysis, validation, writing - review and editing, supervision.

Funding The authors have not declared a specific grant for this research from any funding agency in the public, commercial or not-for-profit sectors.

Competing interests None declared.

Patient consent for publication Not required.

Ethics approval According to the Finnish research legislation and the Finnish National Board on Research Integrity, appointed by the Ministry of Education and Culture, 'The review of the ethics committee is not required for the research of public and published data, registry and documentary data and archive data'. The Ethics Committee of Tampere University Hospital has waived ethical evaluation of all register-based studies in which the participants are not contacted. Institutional permissions were obtained from the chief doctors of each of the participating hospitals to access the hospital discharge register data. Informed consent from patients is not needed when retrospective register data are handled and the participants are not contacted.

Provenance and peer review Not commissioned; externally peer reviewed.

Data availability statement Data are available upon reasonable request. All data relevant to the study are included in the article or uploaded as supplementary information.

Open access This is an open access article distributed in accordance with the Creative Commons Attribution Non Commercial (CC BY-NC 4.0) license, which permits others to distribute, remix, adapt, build upon this work non-commercially, and license their derivative works on different terms, provided the original work is properly cited, appropriate credit is given, any changes made indicated, and the use is non-commercial. See: http://creativecommons.org/licenses/by-nc/4.0/.

ORCID iD

Joonas Kuorikoski http://orcid.org/0000-0002-1768-5822

\section{REFERENCES}

1 World Health Organization (WHO). Coronavirus disease (COVID-19) pandemic. Available: https://www.who.int/emergencies/diseases/ novel-coronavirus-2019 [Accessed 27 Feb 2021].

2 The lancet child \& adolescent health. growing up in the shadow of COVID-19. Lancet Child Adolesc. Heal 2020;4:853.

3 Government Communications Department, Ministry of Education and Culture, Ministry of Social Affairs and Health. Government, in cooperation with the president of the Republic, declares a state of emergency in Finland over coronavirus outbreak, 2020. Available: https://valtioneuvosto.fi/en/-/10616/hallitus-totesi-suomen-olevanpoikkeusoloissa-koronavirustilanteen-vuoksi [Accessed 2 Mar 2021].

4 Finnish Institute for health and welfare. situation update on coronavirus., 2020. Available: https://thl.fi/en/web/infectious- 
diseases-and-vaccinations/what-s-new/coronavirus-covid-19latest-updates/situation-update-on-coronavirus [Accessed 2 Mar 2021].

5 Ministry of Social Affairs and Health Helsinki. Action plan for implementing recommendations and restrictive measures under the hybrid strategy following the first phase of the COVID-19 epidemic publications of the Ministry of social Affairs and health, 2021. Available: https://julkaisut.valtioneuvosto.fi/bitstream/handle/10024/ 163304/STM_2021_26_J.pdf?sequence=1\&isAllowed=y [Accessed 2 Mar 2021].

6 Kuitunen I, Ponkilainen VT, Launonen AP, et al. The effect of national lockdown due to COVID-19 on emergency department visits. Scand J Trauma Resusc Emerg Med 2020;28:114.

7 Tuominen J, Hällberg V, Oksala N, et al. NYU-EDA in modelling the effect of COVID-19 on patient volumes in a Finnish emergency department. BMC Emerg Med 2020;20:97.

8 Sheridan GA, Nagle M, Russell S, et al. Pediatric trauma and the COVID-19 pandemic: a 12-year comparison in a Level-1 trauma center. Hss J 2020:92-6.

9 Baxter I, Hancock G, Clark M. Paediatric orthopaedics in lockdown: a study on the effect of the SARS-Cov-2 pandemic on acute paediatric orthopaedics and trauma. Bone Jt open 2020;1:424-30.

10 Keays G, Friedman D, Gagnon I. Original quantitative research pPediatric injuries in the time of COVID-19. Heal Promot Chronic Dis Prev Canada 2020;40:336-41.

11 Bram JT, Johnson MA, Magee LC, et al. Where have all the fractures gone? the epidemiology of pediatric fractures during the COVID-19 pandemic. J Pediatr Orthop 2020;40:373-9.
12 Nabian MH, Vosoughi F, Najafi F, et al. Epidemiological pattern of pediatric trauma in COVID-19 outbreak: data from a tertiary trauma center in Iran. Injury 2020;51:2811-5.

13 Sugand K, Park C, Morgan C, et al. Impact of the COVID-19 pandemic on paediatric orthopaedic trauma workload in centra London: a multi-centre longitudinal observational study over the "golden weeks". Acta Orthop 2020;91:633-8.

14 Riuttanen A, Ponkilainen V, Kuitunen I, et al. Severely injured patients do not disappear in a pandemic: incidence and characteristics of severe injuries during COVID-19 lockdown in Finland. Acta Orthop 2021;92:249-53.

15 Raitio A, Ahonen M, Jääskelä M, et al. Reduced number of pediatric orthopedic trauma requiring operative treatment during COVID-19 restrictions: a nationwide cohort study. Scand J Surg 2021;110:254-7.

16 Sanford EL, Zagory J, Blackwell J-M, et al. Changes in pediatric trauma during COVID-19 stay-at-home epoch at a tertiary pediatric hospital. J Pediatr Surg 2021;56:918-22.

17 Statistics Finland. Statistics Finland, 2020. Available: https://www. stat.fi/index_en.html [Accessed 2 Mar 2021].

18 Samuel N, Jacob R, Eilon Y, et al. Falls in young children with minor head injury: a prospective analysis of injury mechanisms. Brain Inj : 2015;29:946-50.

19 Hedström EM, Svensson O, Bergström U. Epidemiology of fractures in children and adolescents: increased incidence over the past decade: a population-based study from northern Sweden. Acta Orthop 2010;81.

20 Rennie L, Court-Brown CM, Mok JYQ, et al. The epidemiology of fractures in children. Injury 2007;38:913-22. 